\title{
Quantum optical approach to Bose-Einstein correlations at fixed multiplicities
}

\section{N. Suzuki*}

Department of comprehensive management, Matsumoto University, Matsumoto 390-1295, Japan

E-mail: suzuki@matsu.ac.jp

\section{Biyajima}

Department of physics, Shinshu University, Matsumoto 390-8621, Japan

E-mail: biyajima@azusa.shinshu-u.ac.jp

Data on negatively charged multiplicity distribution and Bose-Einstein correlations of identical particles at fixed multiplicities at $\sqrt{s}=900 \mathrm{GeV}$ in pp collisions are analyzed by a model in the quantum optical approach.

The Seventh Workshop on Particle Correlations and Femtoscopy

September 20 - 242011

University of Tokyo, Japan

\footnotetext{
* Speaker.
} 


\section{Introduction}

In high energy nucleus-nucleus collisions or hadron-hadron collisions, Bose-Einstein correlations of identical particles are considered as one of the possible measures for the space-time domain where identical particles are produced. If multiplicity distribution (MD) and Bose-Einstein correlations (BEC) are constructed from the same observed data sample, some information on BEC would be contained in the MD. Therefore, we can estimate parameters on BEC precisely from the observed MD and BEC at fixed multiplicities.

One of the theoretical approaches to BEC is made on the analogy of the quantum optics [1], where two types of sources, chaotic and coherent are introduced. In Ref.[2], formulae for MD and BEC in semi-inclusive events are derived in the QO approach, and a diagrammatic representation of the cumulants is proposed.

Recently, new data on BEC and MDs in pp collisions are reported from the LHC experiments. In the present paper, MD and $\mathrm{BEC}$ are analyzed by the formulae derived in the $\mathrm{QO}$ approach.

\section{Momentum densities in semi-inclusive events}

The $n$-particle momentum density in semi-inclusive events in the QO approach is defined by,

$$
\rho_{n}\left(p_{1}, \cdots, p_{n}\right)=c_{0}\left\langle\left|f\left(p_{1}\right)\right|^{2} \cdots\left|f\left(p_{n}\right)\right|^{2}\right\rangle_{a}, \quad f(p)=\sum_{i=1}^{M} a_{i} \phi_{i}(p)+f_{c}(p) .
$$

In Eq. 2.1], $c_{0}$ denotes a normalization factor, $f(p)$ is an amplitude composed of that of the $i$-th chaotic source, $\phi_{i}(p)$, and that of the coherent source, $f_{c}(p)$, and $a_{i}$ is a random complex number attached to the $i$-th chaotic source. The number of independent chaotic sources, $M$, is assumed to be infinite[2]. Parenthesis $\langle F\rangle_{a}$ in Eq.(2.1) denotes the average of $F$ over the random number $a_{i}$ with a Gaussian weight [3];

$$
\langle F\rangle_{a}=\left(\prod_{i=1}^{M} \frac{1}{\pi \lambda_{i}} \int \exp \left[-\frac{\left|a_{i}\right|^{2}}{\lambda_{i}}\right] d^{2} a_{i}\right) F
$$

The generating functional (GF) of momentum densities in semi-inclusive events is defined by

$$
Z_{\mathrm{sm}}[h(p)]=c_{0} \sum_{n=0}^{\infty} \frac{1}{n !}\left\langle\left[\int|f(p)|^{2} h(p) \frac{d^{3} p}{E}\right]^{n}\right\rangle_{a},
$$

where $h(p)$ is an arbitrary function. The $n$-particle momentum density and the $n$-th order cumulant in semi-inclusive events are given respectively by

$$
\begin{aligned}
& \rho_{n}\left(p_{1}, \cdots, p_{n}\right)=\left.E_{1} \cdots E_{n} \frac{\delta^{n} Z_{\mathrm{sm}}[h(p)]}{\delta h\left(p_{1}\right) \cdots \delta h\left(p_{n}\right)}\right|_{h(p)=0}, \\
& g_{n}\left(p_{1}, \cdots, p_{n}\right)=\left.E_{1} \cdots E_{n} \frac{\delta^{n} \ln Z_{\mathrm{sm}}[h(p)]}{\delta h\left(p_{1}\right) \cdots \delta h\left(p_{n}\right)}\right|_{h(p)=0} .
\end{aligned}
$$


For a review of semi-inclusive events, see [4]. From Eqs. 2.5] and (2.6), we have an iteration relation for momentum densities,

$$
\begin{aligned}
\rho_{1}\left(p_{1}\right) & =c_{0} g_{1}\left(p_{1}\right)=c_{0}\left[r\left(p_{1}, p_{1}\right)+c\left(p_{1}, p_{1}\right)\right], \\
\rho_{2}\left(p_{1}, p_{2}\right) & =g_{1}\left(p_{1}\right) \rho\left(p_{2}\right)+c_{0}\left\{\left|r\left(p_{1}, p_{2}\right)\right|^{2}+2 \operatorname{Re}\left[r\left(p_{1}, p_{2}\right) c\left(p_{2}, p_{1}\right)\right]\right\}, \\
. \rho_{n}\left(p_{1}, \cdots, p_{n}\right) & =g_{1}\left(p_{1}\right) \rho_{n-1}\left(p_{2}, \cdots, p_{n}\right)+c_{0} g_{n}\left(p_{1}, \cdots, p_{n}\right) \\
& +\sum_{i=1}^{n-2} \sum g_{i+1}\left(p_{1}, p_{j_{1}}, \cdots, p_{j_{i}}\right) \rho_{n-i-1}\left(p_{j_{i+1}}, \cdots, p_{j_{n-1}}\right), \text { for } n \geq 2 .
\end{aligned}
$$

In Eq. (2.6), $r\left(p_{1}, p_{2}\right)$ is a correlation caused by the chaotic sources, $r\left(p_{1}, p_{2}\right)=\sum_{i=1}^{M} \lambda_{i} \phi_{i}\left(p_{1}\right) \phi_{i}^{*}\left(p_{2}\right)$, and $c\left(p_{1}, p_{2}\right)$ is a correlation by the coherent source, $c\left(p_{1}, p_{2}\right)=f_{c}\left(p_{1}\right) f_{c}^{*}\left(p_{2}\right)$.

The second summation on the right hand side of Eq.22.7 indicates that all possible combinations of $\left(j_{1}, \cdots, j_{i}\right)$ and $\left(j_{i+1}, \cdots, j_{n-1}\right)$ are taken from $(2,3, \cdots, n)$.

In order to calculate momentum densities at fixed multiplicity, following equations are defined;

$$
\begin{aligned}
& \rho_{n}^{(k)}\left(p_{1}, \cdots, p_{k}\right)=\frac{1}{(n-k) !} \int \cdots \int \rho_{n}\left(p_{1}, \cdots, p_{k}, p_{k+1}, \cdots, p_{n}\right) \frac{d^{3} p_{k+1}}{E_{k+1}} \cdots \frac{d^{3} p_{n}}{E_{n}} \\
& g_{n}^{(k)}\left(p_{1}, \cdots, p_{k}\right)=\frac{1}{(n-k) !} \int \cdots \int g_{n}\left(p_{1}, \cdots, p_{k}, p_{k+1}, \cdots, p_{n}\right) \frac{d^{3} p_{k+1}}{E_{k+1}} \cdots \frac{d^{3} p_{n}}{E_{n}} .
\end{aligned}
$$

The MD is given by $P(0)=Z_{\mathrm{sm}}[0]=c_{0}$ and

$$
P(n)=\rho_{n}^{(0)}=\frac{(n-k) !}{n !}=\int \cdots \int \rho_{n}^{(k)}\left(p_{1}, \cdots, p_{k}\right) \frac{d^{3} p_{1}}{E_{1}} \cdots \frac{d^{3} p_{k}}{E_{k}} .
$$

For a review on MD, see for example [5]. From Eq. 22.7], we have [2]

$$
\begin{aligned}
\rho_{n}^{(1)}\left(p_{1}\right) & =\sum_{j=1}^{n} j g_{j}^{(1)}\left(p_{1}\right) P(n-j), \quad g_{j}^{(1)}\left(p_{1}\right)=R_{j}\left(p_{1}, p_{1}\right)+\sum_{l=0}^{j-1} T_{l, j-l-1}\left(p_{1}, p_{1}\right), \text { for } n \text { 2.11,0) } \\
\rho_{n}^{(2)}\left(p_{1}, p_{2}\right) & =\sum_{j=1}^{n-1}(n-j) g_{j}^{(1)}\left(p_{1}\right) \rho_{n-j}^{(1)}\left(p_{2}\right)+\sum_{j=2}^{n} g_{j}^{(2)}\left(p_{1}, p_{2}\right) P(n-j), \\
g_{j}^{(2)}\left(p_{1}, p_{2}\right) & =\sum_{l=1}^{j-1} R_{j}\left(p_{1}, p_{2}\right) R_{j-l}\left(p_{2}, p_{1}\right) \\
+ & \sum_{l=0}^{j-2} \sum_{m=0}^{l}\left\{T_{m, l-m}\left(p_{1}, p_{2}\right) R_{j-l-1}\left(p_{2}, p_{1}\right)+R_{j-l-1}\left(p_{1}, p_{2}\right) T_{m, l-m}\left(p_{2}, p_{1}\right)\right\}
\end{aligned}
$$

where, with $R_{0}\left(p_{1}, p_{2}\right)=E_{1} \delta^{3}\left(p_{1}, p_{2}\right)$,

$$
\begin{aligned}
R_{j}\left(p_{1}, p_{2}\right) & =\int r\left(p_{1}, p^{\prime}\right) R_{j-1}\left(p^{\prime}, p_{2}\right) \frac{d^{3} p^{\prime}}{E^{\prime}} \\
T_{j, l}\left(p_{1}, p_{2}\right) & =\iint R_{j}\left(p_{1}, p_{1}^{\prime}\right) c\left(p_{1}^{\prime}, p_{2}^{\prime}\right) R_{l}\left(p_{2}^{\prime}, p_{2}\right) \frac{d^{3} p_{1}^{\prime}}{E_{1}^{\prime}} \frac{d^{3} p_{2}^{\prime}}{E_{2}^{\prime}} .
\end{aligned}
$$




\section{Formulae for MD and BEC}

In the followings, rapidity $y_{i}=\tanh ^{-1}\left(p_{i L} / E_{i}\right)$ and transverse momentum $\boldsymbol{p}_{i T}(i=1,2, \cdots)$ are used. Correlations $r\left(p_{1}, p_{2}\right)$ and $c\left(p_{1}, p_{2}\right)$ are both assumed to be real and parametrized as,

$$
\begin{aligned}
r\left(y_{1}, \boldsymbol{p}_{1 T} ; y_{2}, \boldsymbol{p}_{2 T}\right) & =p_{\mathrm{sm}} \sqrt{\boldsymbol{\rho}\left(y_{1}, \boldsymbol{p}_{1 T}\right) \rho\left(y_{2}, \boldsymbol{p}_{2 T}\right)} \exp \left[-\gamma_{L}(\Delta y)^{2}-\gamma_{T}\left(\Delta \boldsymbol{p}_{T}\right)^{2}\right], \\
c\left(y_{1}, \boldsymbol{p}_{1 T} ; y_{2}, \boldsymbol{p}_{2 T}\right) & =\left(1-p_{\mathrm{sm}}\right) \sqrt{\rho\left(y_{1}, \boldsymbol{p}_{1 T}\right) \rho\left(y_{2}, \boldsymbol{p}_{2 T}\right)}, \\
\rho\left(y_{1}, \boldsymbol{p}_{1 T}\right) & =\left\langle n_{0}\right\rangle \sqrt{\pi / \alpha}(\pi / \boldsymbol{\beta}) \exp \left[-\alpha y_{1}^{2}-\beta \boldsymbol{p}_{1 T}^{2}\right]
\end{aligned}
$$

where $\Delta y=y_{2}-y_{1}$ and $\Delta \boldsymbol{p}_{T}=\boldsymbol{p}_{2 T}-\boldsymbol{p}_{1 T}$. The chaoticity parameter in semi-inclusive events is assumed to be constant, and is denoted by $p_{\mathrm{sm}}\left(=r\left(p_{i}, p_{i}\right) / \rho\left(p_{i}\right)\right)$. As the longitudinal momentum transfer squared, $Q_{L}^{2}=\left(E_{1}-E_{2}\right)^{2}-\left(p_{1 L}-p_{2 L}\right)^{2}$, is approximately written as $Q_{L}^{2} \approx\left\langle m_{T}\right\rangle^{2}(\Delta y)^{2}$ for $|\Delta y|<<1$ with average transverse mass $\left\langle m_{T}\right\rangle, \sqrt{\gamma_{L}} /\left\langle m_{T}\right\rangle$ roughly equal to the longitudinal source size, and $\sqrt{\gamma_{T}}$ is the transverse souce size. Parameter $\left\langle n_{0}\right\rangle$ is related to an average multiplicity. If $p_{\mathrm{sm}}=0$, the MD defined by Eq. 3.1) becomes a Poisson distribution with an average $\left\langle n_{0}\right\rangle$. Parameters $\alpha$ are $\beta$ are related to the width of rapidity distribution and that of $p_{T}$ distribution, respectively.

Then, function $R_{j}\left(y_{1}, \boldsymbol{p}_{1 T}, y_{2}, \boldsymbol{p}_{2 T}\right)$ in Eq.(2.12) is written as,

$$
R_{j}\left(y_{1}, \boldsymbol{p}_{1 T}, y_{2}, \boldsymbol{p}_{2 T}\right)=N_{j} \exp \left[-A_{j}\left(y_{1}^{2}+y_{2}^{2}\right)+2 C_{j} y_{1} y_{2}-U_{j}\left(\boldsymbol{p}_{1 T}^{2}+\boldsymbol{p}_{2 T}^{2}\right)+2 W_{j} \boldsymbol{p}_{1 T} \boldsymbol{p}_{2 T}\right],
$$

where [6, 7

$$
\begin{aligned}
A_{j} & =\frac{r_{2}-r_{1}}{2} \frac{1+\left(r_{1} / r_{2}\right)^{j}}{1-\left(r_{1} / r_{2}\right)^{j}}, \quad C_{j}=\left(r_{2}-r_{1}\right) \frac{\left(r_{1} / r_{2}\right)^{j / 2}}{1-\left(r_{1} / r_{2}\right)^{j}}, \\
U_{j} & =\frac{t_{2}-t_{1}}{2} \frac{1+\left(t_{1} / t_{2}\right)^{j}}{1-\left(t_{1} / t_{2}\right)^{j}}, \quad W_{j}=\left(t_{2}-t_{1}\right) \frac{\left(t_{1} / t_{2}\right)^{j / 2}}{1-\left(t_{1} / t_{2}\right)^{j}}, \\
N_{j} & =\frac{r_{2}{ }^{1 / 2} t_{2}}{\pi^{3 / 2}}\left(\frac{p_{\mathrm{sm}}\left\langle n_{0}\right\rangle \alpha^{1 / 2} \beta}{r_{2}{ }^{1 / 2} t_{2}}\right)^{j}\left\{\frac{1-\left(r_{1} / r_{2}\right)}{1-\left(r_{1} / r_{2}\right)^{j}}\right\}^{1 / 2} \frac{1-\left(t_{1} / t_{2}\right)}{1-\left(t_{1} / t_{2}\right)^{j}}, \\
r_{1} & =\frac{1}{2}\left[\alpha+2 \gamma_{L}-\sqrt{\alpha^{2}+4 \alpha \gamma_{L}}\right], \quad r_{2}=\frac{1}{2}\left[\alpha+2 \gamma_{L}+\sqrt{\alpha^{2}+4 \alpha \gamma_{L}}\right] \\
t_{1} & =\frac{1}{2}\left[\beta+2 \gamma_{T}-\sqrt{\beta^{2}+4 \beta \gamma_{T}}\right], \quad t_{2}=\frac{1}{2}\left[\beta+2 \gamma_{T}+\sqrt{\beta^{2}+4 \beta \gamma_{T}}\right] .
\end{aligned}
$$

The MD in the QO approach is written as [7],

$$
P(n)=\frac{1}{n} \sum_{j=1}^{n}\left(\Delta_{j}^{(R)}+j \Delta_{j-1}^{(S)}\right) P(n-j),
$$

where

$$
\begin{aligned}
& \Delta_{j}^{(R)}=p_{\mathrm{sm}}\left\langle n_{0}\right\rangle\left(\frac{p_{\mathrm{sm}}\left\langle n_{0}\right\rangle \sqrt{\alpha} \beta}{\sqrt{r_{2}} t_{2}}\right)^{j-1} \frac{1-\sqrt{r_{1} / r_{2}}}{1-\left(r_{1} / r_{2}\right)^{j / 2}}\left\{\frac{1-\sqrt{t_{1} / t_{2}}}{1-\left(t_{1} / t_{2}\right)^{j / 2}}\right\}^{2}, \\
& \Delta_{j-1}^{(S)}=\left(1-p_{\mathrm{sm}}\right)\left\langle n_{0}\right\rangle\left(\frac{p_{\mathrm{sm}}\left\langle n_{0}\right\rangle \sqrt{\alpha} \beta}{\sqrt{r_{2}} t_{2}}\right)^{j-1}\left\{\frac{1-\left(r_{1} / r_{2}\right)}{1-\left(r_{1} / r_{2}\right)^{j}}\right\}^{1 / 2} \frac{1-\left(t_{1} / t_{2}\right)}{1-\left(t_{1} / t_{2}\right)^{j}} .
\end{aligned}
$$

As can be seen from the above equations, the MD contains four parameters, $p_{\mathrm{sm}},\left\langle n_{0}\right\rangle, h_{L}=\gamma_{L} / \alpha$ and $h_{T}=\gamma_{T} / \beta$. The inclusive one-particle rapidity distribution is given by

$$
\rho^{(1)}(y)=\sum_{n=1}^{n_{\max }} \rho_{n}^{(1)}(y), \quad \rho_{n}^{(1)}(y)=\int \rho_{n}^{(1)}\left(y, \boldsymbol{p}_{T}\right) d^{2} \boldsymbol{p}_{T} .
$$


The Bose-Einstein correlation function $C_{n}^{(2)}(\Delta y)$ at $n$-particle events is defined as

$$
C_{n}^{(2)}(\Delta y)=\frac{n P(n)}{n-1} \frac{\iiint \rho_{n}^{(2)}\left(y_{1}, \boldsymbol{p}_{1 T}, y_{1}+\Delta y, \boldsymbol{p}_{1 T}+\Delta \boldsymbol{p}_{T}\right) d y_{1} d^{2} \boldsymbol{p}_{1 T} d^{2} \Delta \boldsymbol{p}_{T}}{\iiint \rho_{n}^{(1)}\left(y_{1}, \boldsymbol{p}_{1 T}\right) \rho_{n}^{(1)}\left(y_{1}+\Delta y, \boldsymbol{p}_{1 T}+\Delta \boldsymbol{p}_{T}\right) d y_{1} d^{2} \boldsymbol{p}_{1 T} d^{2} \Delta \boldsymbol{p}_{T}} .
$$

\section{Analysis of experimental data}

The MD for negatively charged particles observed in the pseudo-rapidity region $|\eta|<1$ in pp collisions at $\sqrt{s}=900 \mathrm{GeV}[8]$ is analyzed by Eq. 3.1]. It is constructed from the even prongs of observed charged MD. At first, it is analyzed with four parameters, $p_{\mathrm{sm}},\left\langle n_{0}\right\rangle, h_{L}$ and $h_{T}$. The result becomes that $h_{T} \approx 0$. Therefore, the data is re-analyzed with three parameters under the condition that $h_{T}=0\left(\gamma_{T}=0\right)$. Estimated parameters are shown in Table 1 and comparison of the calculated result with the observed MD is shown in Fig 1

\begin{tabular}{cccc}
\hline$p_{\mathrm{sm}}$ & $\left\langle n_{0}\right\rangle$ & $h_{L}$ & $\chi_{\min }^{2} / n . d . f$ \\
\hline $0.670 \pm 0.052$ & $1.100 \pm 0.10$ & $0.351 \pm 0.294$ & $41.1 /(22-3)$ \\
\hline
\end{tabular}

Table 1: Estimated parameters in the analysis of negatively charged MD observed in pp collisions «8].

In our calculations, transverse momentum is integrated. Therefore, parameter $\beta$ is not included in Eqs. (3.2) and (3.3). Calculated result on inclusive one-particle rapidity distributions is shown in Fig 2 Calculated result on Bose-Einstein correlation functions at fixed multiplicities as a function of $\Delta y$ is shown in Fig 3 That of Bose-Einstein correlation functions at $\Delta y=0$ is compared with the data at $Q_{\text {inv }}=0$ in Fig 4 The data is presented in four $p_{T}$ range [8]. Therefore we use the average value of four cases.

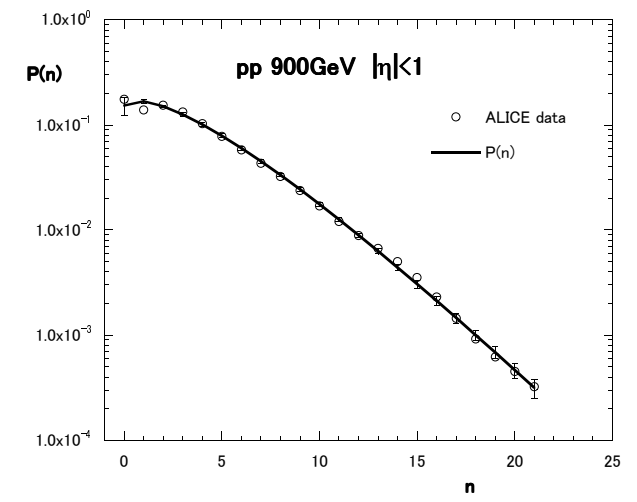

Figure 1: Analysis of negatively charged $\mathrm{MD}$ in $|\eta|<1$ in pp collisions [8] by Eq. 3.1.

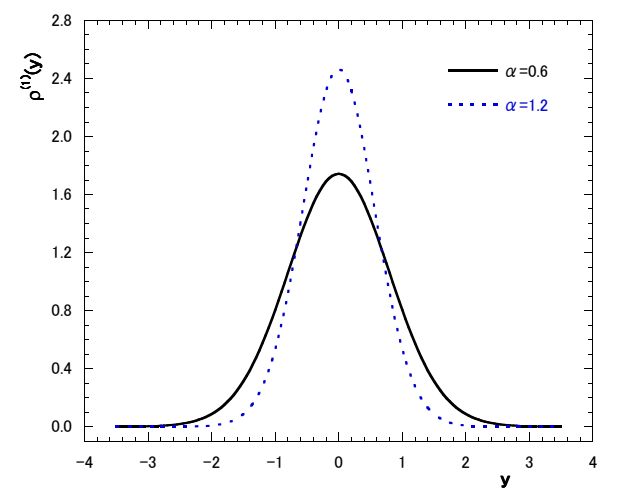

Figure 2: Inclusive one-particle rapidity distributions calculated with $\alpha=0.6$ and 1.2 by Eq. 3.2.

\section{Summary}

The observed MD and BEC at $\Delta y=0\left(Q_{\text {inv }}=0\right)$ are analyzed by our model in the QO approach. In our formulation, six parameters, $p_{\mathrm{sm}},\left\langle n_{0}\right\rangle, h_{L}=\gamma_{L} / \alpha, h_{T}=\gamma_{T} / \beta, \alpha$ and $\beta$ are con- 


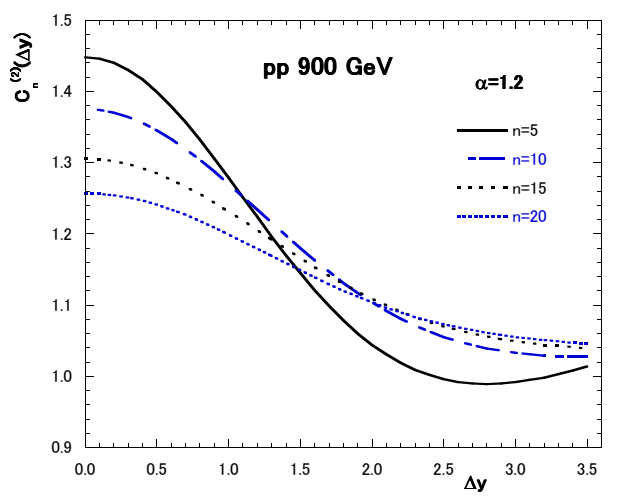

Figure 3: Bose-Einstein correlation function $C_{n}^{(2)}(\Delta y)$ calculated with $\alpha=1.2$ by Eq. 3.3.

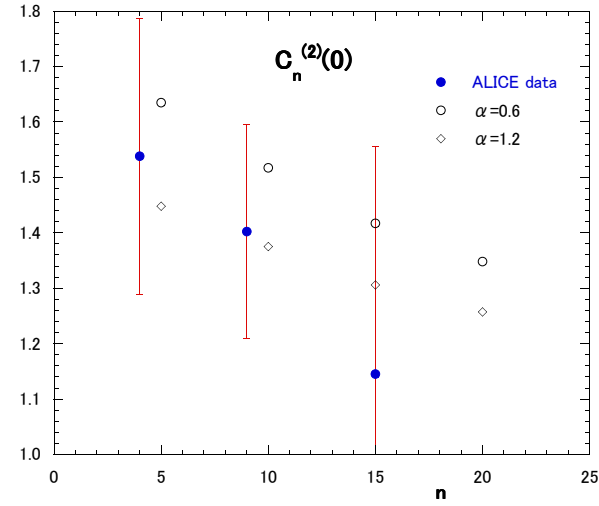

Figure 4: Comparison of $C_{n}^{(0)}(0)$ calculated with $\alpha=0.6$ and 1.2 with the data [9].

tained. In the present analysis, parameter $\gamma_{T}$ becomes effectively zero and $\beta$ is not included in Eq.(3.3). Therefore, only parameter $\alpha$ is effective to the analysis of BEC. As can be seen from Fig 4 calculated result of $C_{n}^{(2)}(0)$ for $0.6 \leq \alpha \leq 1.2$ would not be inconsistent with the observed data at $\Delta y=0\left(Q_{\mathrm{inv}}=0\right)$. If the $\mathrm{MD}$ and $\mathrm{BEC}$ at fixed multiplicities are constructed from the same data sample, we would obtain more precise information on the production region of like-sign charged particles in the final states of hadronic interactions.

\section{References}

[1] R. J. Glauber, Coherent and incoherent ststes of the radiation field, Phys. Rev. 131, 2766(1963).

[2] N.Suzuki and M. Biyajima, Multiplicity dependence of identical particle correlations in the quantum optical approach, Phys. Rev. C60, 034903(1999).

[3] M. Biyajima, O. Miyamura and T. Nakai, An analysis of identical pion distributions by means of superposed coherent and chaotic emission, Proceedings of the Multiparticle Dynamics, Hakone, Japan, 1978 (PIFP, Kyoto Univ., Japan, 1978), p.139.

[4] W.Kittel and E. A. De Wolf, Soft Multihadron Dynamics, World Scientific, Singapore, 2005.

[5] I. M. Dremin and J. W. Gary, Hadron Multiplicities, Phys. Rep. 349, 301(2001) [hep-th0004215].

[6] T. Csörgô and J. Zimanyi, Analytic solution of the pion-laser model, Phys. Rev. Lett. 80, 916(1998); J. Zimanyi and T. Csörgó, Multi-Boson Correlation Using Wave-Packets, Heavy Ion Physics, 9 , 241(1999).

[7] N. Suzuki, M. Biyajima and T. Mizoguchi, Estimation of multi-particle correlation from multiplicity distribution observed in relativistic heavy ion collisions, Physics of Particles and Nuclei Letters, $\mathbf{8}$, 1007(2011).

[8] K. Aamodt, et al. (ALICE Collaboration), Charged-particle multiplicity measurement in proton-proton collisions at $\sqrt{s}=0.9$ and 2.36 TeV with ALICE at LHC, Eur. Phy. J. C68,89(2010).

[9] K. Aamodt, et al. (ALICE Collaboration), Two-pion Bose-Einstein correlations in pp collisions at $\sqrt{s}=900$ GeV, Phys. Rev. D82, 052001(2010). 